\title{
Prevalência de Doenças Tireoidianas Auto-Imunes em Pacientes com Lúpus Eritematoso Sistêmico
}

\begin{abstract}
RESUMO
Introdução: As doenças tireoidianas auto-imunes têm sido associadas com inúmeras desordens reumatológicas, incluindo o lúpus eritematoso sistêmico (LES). Objetivo: Avaliar a prevalência de alterações tireoidianas em pacientes com LES. Métodos: Estudo de coorte transversal comparando prevalência de alterações tireoidianas em 106 pacientes com LES e grupo-controle de 102 pacientes. Os pacientes foram submetidos à avaliação clínica e laboratorial, com dosagens de anticorpos anti-TPO e anti-Tg, TSH, T4 livre e T3. Resultados: Detectou-se aumento de prevalência de hipotireoidismo subclínico $(11 \%)$ e clínico $(13 \%)$ no grupo de pacientes com LES, comparados a $7 \%$ e $1 \%$, respectivamente, no grupo-controle $(p<0,001)$. Não houve diferença de prevalência de hipertireoidismo entre os grupos comparados. A positividade de anticorpos antitireoidianos foi maior no grupo de LES $(23 \%$ versus $19 \%, p=0,025)$. O risco relativo de pacientes com LES apresentarem alterações tireoidianas foi de 1,60. Conclusão: As alterações tireoidianas são mais freqüentes em pacientes com LES que na população geral. Arq Bras Endocrinol Metab 2008; 52/3:531-536)
\end{abstract}

Descritores: Lúpus eritematoso sistêmico; Hipotireoidismo; Hipertireoidismo; Doenças auto-imunes; Glândula tireóide

\begin{abstract}
Prevalence of Thyroid Autoimmune Disease in Patients with Systemic Lupus Erythematosus.

Introduction: Thyroid autoimmune diseases have been associated with a variety of rheumatologic diseases, including systemic lupus erythematosus (SLE). Objective: To estimate the prevalence of thyroid autoimmune diseases in patients with SLE. Methods: Transversal study to compare the prevalence of thyroid disorders in 106 patients with SLE and a control group of 102 patients. The patients were submitted to a clinical and a laboratorial evaluation; the tests included antiperoxidase and antithyroglobulin antibodies, TSH, free T4 and T3. Results: We detected high prevalence of subclinical hypothyroidism $(11 \%)$ and clinical hypothyroidism $(13 \%)$ in the group of patients with SLE when they were compared to the control group, $7 \%$ and $1 \%$, respectively $(p<0,001)$. The hyperthyroidism occurred in a same frequency in the two groups. The positivity of antithyroid antibodies was higher in the group with SLE $(23 \%$ versus $19 \%, p=0,025)$. The relative risk of the patients with SLE to develop thyroid dysfunctions was 1,6. Conclusion: The thyroid disorders were more prevalent in patients with LES than in general population. Arq
\end{abstract} Bras Endocrinol Metab 2008; 52/3:531-536)

Keywords: Systemic lupus erythematosus; Hypothyroidism; Hyperthyroidism; Autoimmune diseases; Thyroid gland artigo original

\author{
Daniela P. P. Oliveira Viggiano
}

NILZIO ANTÔNIO dA SILVA

Ana C. O. e Silva Montandon

Vitalina de Souza Barbosa

Departamento de Clínica

Médica do Serviço de

Endocrinologia e Metabologia

do Hospital das Clínicas da

Universidade Federal de Goiás

(HC-UFG), Goiânia, GO, Brasil

(DPPOV); Departamento de

Clínica Médica do Serviço de

Reumatologia do HC-UFG,

Goiânia, GO, Brasil (NAS, VSB);

Centro de Reabilitação e

Recuperação de Goiás (Crer-

GO), Goiânia, GO, Brasil

(ACOSM)

Trabalho realizado no

Departamento de Clínica

Médica, Serviço de

Reumatologia do Hospital das

Clínicas, Faculdade de Medicina da Universidade Federal de Goiás.

Recebido em 04/12/2007

Aceito em 28/01/2008 


\section{INTRODUÇÃO}

s dOENÇAS AUTO-IMUNES SÃo síndromes clínicas distintas caracterizadas por várias alterações na resposta imune normal, com perda da tolerância para constituintes do próprio hospedeiro (1).

São divididas em sistêmicas e órgão-específicas. Dentre as doenças auto-imunes inflamatórias sistêmicas estão incluídas a artrite reumatóide, o lúpus eritematoso sistêmico (LES), a dermatomiosite, a polimiosite, a esclerose sistêmica, as vasculites e a síndrome de Sjögren (SS). Em relação à auto-imunidade órgão-específica, na qual órgãos específicos são alvo de dano auto-imune, destacamse o diabetes melito tipo 1, a doença de Graves (DG), a tireoidite de Hashimoto ( $\mathrm{TH})$, a anemia perniciosa, a doença de Addison e a miastenia gravis (2).

O LES é doença inflamatória crônica, multissistêmica, de etiologia desconhecida e de natureza auto-imune, caracterizada pela presença de diversos auto-anticorpos. Evolui com manifestações clínicas polimórficas, com períodos de exacerbações e remissões. $\mathrm{O}$ desenvolvimento da doença está ligado a predisposição genética e fatores ambientais, como luz ultravioleta e alguns medicamentos, como hidralazina, procainamida e hidantoinatos. Sua patogenia é caracterizada por lesões teciduais provocadas por reações imunológicas, com formação de complexos antígeno-anticorpo e fixação de complemento, além da citotoxicidade mediada por anticorpos (antihemácias, antiplaquetários e antilinfócitos) (3).

As doenças tireoidianas auto-imunes incluem principalmente a TH e a DG (4), sendo associadas com inúmeras desordens reumatológicas sistêmicas, incluindo LES $(5,6)$, SS e arterite de células gigantes (7). Essa associação parece estar relacionada a alelos específicos do complexo maior de histocompatibilidade, sendo os mais comumente relacionados HLA DR2, DR3 e DR8 (8).

Alguns estudos sugerem que a doença tireoidiana auto-imune é mais comum nos portadores de LES que na população geral, mesmo naqueles sem doença tireoidiana clínica (8-20). Se o LES é um fator de risco independente para anormalidades tireoidianas autoimunes ou apenas compartilha o mesmo grupo de risco, ainda é assunto controvertido (9). Não obstante o LES ser doença auto-imune sistêmica e as doenças tireoidianas auto-imunes serem órgão-específicas, a etiopatogenia em comum, que é a auto-imunidade, poderia justificar a inter-relação.

Clínicos que tratam pacientes com LES têm de estar atentos aos sinais e sintomas das doenças tireoidia- nas auto-imunes. As manifestações podem ser subclínicas e agravar os sintomas do LES, especialmente na fase inicial da doença, sendo o diagnóstico precoce fator relevante para o melhor prognóstico do tratamento.

Pesquisas para avaliação da associação do LES e desordens tireoidianas são necessárias para definir o benefício do rastreio bioquímico da função tireoidiana em pacientes com LES. Como os dados são conflitantes e a prevalência da associação entre o LES e as doenças tireoidianas auto-imunes é variável dependendo do local de estudo, o presente trabalho teve como objetivos: avaliar a prevalência de alterações tireoidianas em pacientes com LES, comparados a um grupo-controle pareado por idade e sexo; caracterizar a prevalência do hipotireoidismo e do hipertireoidismo clínico e suas alterações subclínicas, bem como a prevalência da positividade dos anticorpos antiTPO e anti-Tg nos pacientes com diagnóstico prévio de LES no Hospital das Clínicas da Faculdade de Medicina da Universidade Federal de Goiás (HC-FM/UFG).

\section{PACIENTES E MÉTODOS}

Estudo de coorte transversal de pacientes do Sistema Único de Saúde (SUS) acompanhados no Serviço de Reumatologia do HC-FM/UFG.

O tamanho da amostra foi calculado de maneira consensual, não-probabilística, com amostra representativa de, no mínimo, 100 pacientes analisados durante um período definido, perfazendo um total mínimo de $25 \%$ dos pacientes lúpicos acompanhados no ambulatório.

No período de maio de 2006 a junho de 2007, foram avaliados 106 pacientes com diagnóstico prévio de LES, segundo critérios de classificação do Colégio Americano de Reumatologia (21), e que estavam em acompanhamento regular, constituindo o grupo de pacientes com LES. O grupo-controle foi constituído por 102 voluntários não-lúpicos e sem disfunção tireoidiana prévia, provenientes do Ambulatório de Clínica Médica do HC-FM/UFG.

Os critérios de inclusão do grupo de pacientes com LES foram: idade superior ou igual a 18 anos; diagnóstico prévio de LES, segundo critérios de classificação do Colégio Americano de Reumatologia (21); acompanhamento no Serviço de Reumatologia do HC-FM/ UFG; e assinatura do Termo de Consentimento Livre e Esclarecido (TCLE) do estudo. No grupo-controle, foram incluídos indivíduos não-lúpicos e sem disfunção tireoidiana prévia, provenientes do Ambulatório de Clí- 
nica Médica do HC-FM/UFG, que concordaram em participar do presente estudo.

Foram excluídas pacientes gestantes e as que engravidaram durante a avaliação, pacientes em uso de drogas que interferem na função tireoidiana (amiodarona e interferon), com idade inferior a 18 anos e os que estavam participando de outros estudos clínicos.

O grupo selecionado foi avaliado em consulta médica no ambulatorial, seguindo as normas preestabelecidas, sendo preenchida ficha de avaliação padronizada. O protocolo para registro dos casos incluiu dados demográficos, medicações utilizadas, tempo de evolução da doença e grau de atividade clínica da doença.

Durante a consulta médica, foi analisada, por meio de anamnese clínica, a presença de sintomas clínicos de hipotireoidismo e hipertireoidismo. O exame físico foi realizado avaliando-se consistência e volume tireoidiano à palpação, medida de circunferência do pescoço em centímetros, presença de mixedema facial e pré-tibial, freqüência cardíaca (FC) em batimentos por minuto (bpm).

Os pacientes incluídos na presente pesquisa foram submetidos à coleta sangüínea para dosagens hormonais de T4 livre, T3, TSH, anti-Tg, anti-TPO, que foram realizadas no Laboratório de Análises Clínicas do HC-UFG por quimioluminescência, utilizando-se estojos comerciais da DPC (Diagnostics Products Corporation - Los Angeles, EUA) e sistema de automação IMULLITE 2000. O TRAb foi dosado somente nos pacientes com quadro clínico e laboratorial de hipertireoidismo, por radioimunoensaio (RSR Limited for Autoimmune Diagnostics, UK). Os valores de referência (VR) foram definidos pelo método laboratorial utilizado e por intervalos de normalidade: T4 livre (VR: 0,8 a 1,9 mg/dl), T3 (VR: 82 a 179 ng/dl), TSH (VR: 0,4 a $4,0 \mu \mathrm{UI} / \mathrm{ml})$, anti-TPO (0 a 50 anos VR: 0 a $34 \mathrm{UI} / \mathrm{ml}$; 50 anos - VR: 0 a $100 \mathrm{UI} / \mathrm{ml}$ ), anti- $\mathrm{Tg}(0$ a 50 anos - VR: 0 a $40 \mathrm{UI} / \mathrm{ml}$; $>50$ anos - VR: 0 a $80 \mathrm{UI} / \mathrm{ml}$ ) e TRAb (VR: 0\% a 10\%).

O protocolo do estudo foi analisado e aprovado pelo Comitê de Ética em Pesquisa Médica Humana e Animal do HC/UFG. Todos os pacientes foram informados sobre o objetivo do estudo, exames a serem realizados e sua segurança e, após esclarecimento de dúvidas, assinaram TCLE.

As doenças tireoidianas auto-imunes foram definidas pela dosagem de anticorpo anti-TPO maior que $34 \mathrm{UI} /$ $\mathrm{ml} \mathrm{em} \mathrm{pacientes} \mathrm{com} \mathrm{até} 50$ anos e maior que $100 \mathrm{UI} /$ $\mathrm{ml}$ em pacientes com mais de 50 anos, e/ou anti-Tg maior que $40 \mathrm{UI} / \mathrm{ml} \mathrm{em} \mathrm{paciente} \mathrm{com} \mathrm{até} 50$ anos e maior que $80 \mathrm{UI} / \mathrm{ml} \mathrm{em} \mathrm{pacientes} \mathrm{com} \mathrm{mais} \mathrm{de} 50$ anos, ou TRAb maior que $10 \%$. O hipotireoidismo primário consistiu de valores de TSH maiores que $4,0 \mu \mathrm{UI} / \mathrm{ml}$ com T4 livre menor que $0,80 \mathrm{mg} / \mathrm{dl}$ ou T3 menor que $82 \mathrm{ng} / \mathrm{dl}$, associado a sinais e sintomas clínicos de hipotireoidismo. Hipertireoidismo foi considerado na presença de supressão de TSH com valores menores que 0,4 $\mu \mathrm{UI} / \mathrm{ml}$ com T4 livre acima de $1,9 \mathrm{mg} / \mathrm{dl}$ e/ou T3 maior que $179 \mathrm{ng} / \mathrm{dl}$, e sintomas clínicos de hipertireoidismo. Foram consideradas como disfunções tireoidianas subclínicas anormalidades no TSH, com níveis de T4 livre e T3 normais, e ausência de sinais e sintomas clínicos de doença tireoidiana (22-25).

Todos os participantes do grupo de pacientes com LES e do grupo-controle, que apresentaram qualquer alteração tireoidiana durante a pesquisa, foram encaminhados ao Serviço de Endocrinologia e Metabologia do HC para tratamento e seguimento adequado.

Para a comparação estatística dos parâmetros de função tireoidiana entre os pacientes com LES e do grupocontrole, foram utilizados testes paramétricos $t$ de Student, análise de variância e Tukey; e para dados cuja distribuição não foi normal, não-paramétricos qui-quadrado, análise de resíduo, binomial, teste exato de Fisher e Friedman. O valor de $\mathrm{p}$ para rejeição da hipótese de nulidade foi fixado em $0,05(\alpha=5 \%)$ e intervalo de confiança de $95 \%$.

A análise estatística foi realizada do NCSS (Number Crunching Statistical Software) 2004, Statistical Package for the Social Sciences (SPSS) for Windows, versão 10.0, da SPSS, Inc (EUA) e Epi Info versão 3.3.2.

\section{RESULTADOS}

O total de 106 pacientes com diagnóstico prévio de LES foi avaliado (4 homens e 102 mulheres), com mediana de idade de 38 anos e 6 meses (mínimo: 18 anos, máximo: 77 anos). O grupo-controle foi constituído por 102 indivíduos sem LES, com mediana de idade de 41 anos e 5 meses (mínimo: 18 anos e máximo: 72 anos), sendo 3 homens e 99 mulheres. O grupo de pacientes com LES apresentou tempo médio de duração da doença de 8 anos e 11 meses (mínimo: 1 ano, máximo: 34 anos); $87 \%$ dos pacientes estavam em uso de drogas imunossupressoras e $26 \%$ com doença inativa (Tabela 1 ).

No que diz respeito às alterações clínicas e laboratoriais da avaliação tireoidiana nos grupos estudados, detectou-se aumento de prevalência de concentrações elevadas de TSH $(\mathrm{p}=0,001)$, diminuição de concentra- 
ção de T4 livre $(\mathrm{p}=0,01)$ e T3 $(\mathrm{p}=0,007)$ no grupo de pacientes com LES, em comparação ao controle.

De acordo com a classificação das alterações tireoidianas, encontrou-se $11 \%$ de hipotireoidismo subclínico e $13 \%$ de hipotireoidismo clínico nos pacientes com LES, comparados a $6 \%$ e $1 \%$ no grupo-controle, respectivamente $(\mathrm{p}<0,001)$ (Tabela 2$)$.
A prevalência de hipertireoidismo foi semelhante no grupo de pacientes com LES e no controle $(\mathrm{p}=$ 0,47 e $\mathrm{p}=0,26$, respectivamente). Todos os casos de hipertireoidismo apresentaram TRAb negativos, e 2 pacientes com hipertireoidismo do grupo LES apresentaram positividade para anticorpos anti-TPO e anti-Tg (Tabela 2).

Tabela 1. Análise das variáveis epidemiológicas dos pacientes com LES e do grupo-controle.

\begin{tabular}{|c|c|c|c|c|c|}
\hline \multirow{2}{*}{ Variáveis clínicas } & \multicolumn{2}{|l|}{ LES $(N=106)$} & \multicolumn{3}{|l|}{ Controle $(\mathrm{N}=102)$} \\
\hline & Mediana (mín.-máx.) & $\mathbf{N}(\%)$ & Mediana (mín.-máx.) & $\mathbf{N}(\%)$ & $p$ \\
\hline Idade (anos; meses) & $38,6(18-77)$ & & $41,5(18-72)$ & & 0,09 \\
\hline \multicolumn{6}{|l|}{ Sexo } \\
\hline masculino & & $4(4,0)$ & & $3(3,0)$ & \multirow{2}{*}{1,0} \\
\hline feminino & & $102(96,0)$ & & $99(97,0)$ & \\
\hline \multicolumn{6}{|l|}{ Uso de imunossupressores } \\
\hline não & & $14(13,0)$ & & & \\
\hline $\operatorname{sim}$ & & $92(87,0)$ & & & \\
\hline $\begin{array}{l}\text { Tempo de diagnóstico } \\
\text { (anos; meses) }\end{array}$ & $8,11(1-34)$ & & & & \\
\hline \multicolumn{6}{|l|}{ Atividade da doença } \\
\hline ativa & & $78(74,0)$ & & & \\
\hline inativa & & $28(26,0)$ & & & \\
\hline
\end{tabular}

Tabela 2. Prevalência das alterações tireoidianas em pacientes com LES e no grupo-controle.

\begin{tabular}{|c|c|c|c|c|c|}
\hline \multirow{2}{*}{$\begin{array}{l}\text { Alteração tiroidiana } \\
\text { Classificação }\end{array}$} & \multicolumn{2}{|c|}{$\begin{array}{c}\text { LES } \\
(N=106)\end{array}$} & \multicolumn{2}{|c|}{$\begin{array}{l}\text { Controle } \\
(\mathrm{N}=102)\end{array}$} & \multirow[b]{2}{*}{ p } \\
\hline & $\mathbf{N}$ & $\%$ & $\mathbf{N}$ & $\%$ & \\
\hline Sem alterações & 61 & 58,0 & 75 & 74,0 & $<0,001$ \\
\hline Hipotireoidismo subclínico e anticorpos negativos & 10 & 9,0 & 2 & 2,0 & 0,021 \\
\hline Hipotireoidismo subclínico e anticorpos positivos & 2 & 2,0 & 4 & 4,0 & 0,314 \\
\hline Hipotireoidismo clínico e anticorpos negativos & 3 & 3,0 & - & 0,0 & 0,134 \\
\hline Hipotireoidismo clínico e anticorpos positivos & 11 & 10,0 & 1 & 1,0 & 0,003 \\
\hline Hipotireoidismo com anticorpos negativos & 1 & 1,0 & 2 & 2,0 & 0,478 \\
\hline Hipotireoidismo com anticorpos positivos & 2 & 2,0 & - & 0,0 & 0,264 \\
\hline $\begin{array}{l}\text { TSH normal e alterações em T3 e/ou T4 } \\
\text { com anticorpos negativos }\end{array}$ & 5 & 5,0 & 0 & 0,0 & 0,034 \\
\hline Apenas alterações nos anticorpos tiroidianos & 9 & 8,0 & 14 & 13,0 & 0,151 \\
\hline Bócio multinodular e nodular & 2 & 2,0 & 4 & 4,0 & 0,667 \\
\hline Hipotireoidismo subclínico e clínico & 26 & 24,0 & 7 & 7,0 & 0,134 \\
\hline Hipotireoidismo subclínico & 12 & 11,0 & 6 & 6,0 & $<0,001$ \\
\hline Hipotireoidismo clínico & 14 & 13,0 & 1 & 1,0 & $<0,001$ \\
\hline
\end{tabular}

$\mathrm{P}<0,05$; LES = lúpus eritematoso sistêmico; $\mathrm{N}$ = número de pacientes; Qui-quadrado, teste exato de Fisher, binomial 
Em relação à positividade de anticorpos tireoidianos, observou-se maior prevalência nos pacientes com LES comparados ao grupo-controle $(23 \%$ versus $19 \%, \mathrm{p}=$ $0,025)$. A presença de positividade para o anticorpo antiTg foi maior no grupo de pacientes com LES (20\% versus $8 \%, \mathrm{p}<0,001)$, bem como a positividade para ambos os anticorpos ( $12 \%$ versus $6 \%, \mathrm{p}<0,001)$ (Tabela 3 ).

A somatória de todas as alterações tireoidianas foi significativamente maior no grupo LES, $45 \%$ versus $27 \%$ no grupo-controle $(\mathrm{p}<0,001)$, sendo o risco relativo de pacientes com LES apresentarem alterações tireoidianas de 1,60 com intervalo de confiança de $95 \%$ de $1,08-2,37$.

Tabela 3. Prevalência de positividade dos anticorpos tireoidianos em pacientes com LES e do grupo-controle.

\begin{tabular}{lccc}
\hline Anticorpos tireoidianos & $\begin{array}{c}\text { LES } \\
\text { N (\%) }\end{array}$ & $\begin{array}{c}\text { Controles } \\
\text { N (\%) }\end{array}$ & p \\
Anticorpos tireoidianos & $24(23)$ & $19(19)$ & 0,025 \\
\hline Anti-TPO & $16(15)$ & $16(16)$ & 0,906 \\
Anti-Tg & $21(20)$ & $8(8)$ & $<0,001$ \\
Anti-TPO e Anti-Tg & $13(12)$ & $6(6)$ & $<0,001$
\end{tabular}

$\mathrm{P}<0,05 ; \mathrm{LES}=$ lúpus eritematoso sistêmico; $N=$ número de pacientes; Anti-TPO = anticorpo antiperoxidase; $\mathrm{Anti-Tg}=$ anticorpo antitireoglobulina

\section{DISCUSSÃo}

Embora seja bem conhecida a associação de doenças auto-imunes sistêmicas e órgão-específicas, a presença de alteração tireoidiana em pacientes com LES ainda é motivo de controvérsia, e como referido anteriormente, pode ser um achado coincidente, por atingirem o mesmo grupo de risco. Os resultados do presente estudo demonstram que pacientes com LES, quando comparados a grupo-controle, apresentam maior prevalência de positividade dos anticorpos tireoidianos, hipotireoidismos clínico e subclínico, indicando que a prevalência de doença auto-imune da tireóide está aumentada nos pacientes com LES.

A idade dos pacientes com alterações tireoidianas foi semelhante entre o grupo de pacientes com LES e o grupo-controle $(\mathrm{p}=0,09)$, o que pode sugerir que o aumento de prevalência das alterações tireoidianas no grupo com LES não seja decorrente do fato coinciden- te por atingir mulheres na mesma faixa etária e, sim, um fator independente.

Os mecanismos de coexistência de doença auto-imune da tireóide e LES são desconhecidos, entretanto diversos mecanismos podem contribuir para esta associação. A auto-reatividade das células $\mathrm{T}$, que causa dano celular tireoidiano, e a ativação das células $\mathrm{B}$ policlonais no LES, podem induzir tireoidite e LES no mesmo paciente (15).

Em 1961, o primeiro caso de LES e TH foi relatado (9). Essas enfermidades acometem pacientes jovens, do sexo feminino, e parecem estar relacionadas a alelos específicos do complexo maior de histocompatibilidade, sendo os mais comumente relacionados, o HLA DR2, o HLA DR3 e o HLA DR8 (8).

Em trabalhos subseqüentes, o aumento da prevalência de alterações tireoidianas em pacientes com LES também foi encontrado $(8,11-13,17)$.

A predominância do hipotireoidismo em relação ao hipertireoidismo nos pacientes com LES foi um achado coincidente com os encontrados por Miller e cols. (11), Park e cols. (15) e Kakehasi e cols. $(18,26)$.

Kakehasi e cols. (26) encontraram alteração tireoidiana em $17 \%$ dos pacientes com LES, todos do sexo feminino, porém, ao contrário de nosso estudo, a alteração mais prevalente foi de hipotireoidismo subclínico (10\%), seguida pelo hipotireoidismo clínico (4\%) e o hipertireoidismo (2\%). Esses dados sugerem que as alterações tireoidianas podem apresentar-se de maneira variável dependendo do local, da população estudada e da metodologia empregada.

Estudo recente de Reuven e cols. (20), em 2007, detectou dados semelhantes aos nossos, com prevalência de $11,6 \%$ de hipotireoidismo nos pacientes com LES comparados a $1,9 \%$ no grupo-controle.

Em pesquisas anteriores, Kausman e Isenberg (16)e Magaro e cols. (13) encontraram 21\% e 45,5\% de positividade para os anticorpos tireoidianos, respectivamente.

Em nosso grupo foi demonstrada a prevalência total de positividade para anticorpos tireoidianos de $23 \%$ no grupo de pacientes com LES e de $19 \%$ no grupo-controle $(\mathrm{p}=0,025)$.

A maior prevalência de pacientes com positividade somente para anticorpo anti-Tg (e anti-TPO negativo), bem como a maior prevalência de pacientes com positividade para ambos os anticorpos no grupo com LES, pode sugerir que para este grupo de pacientes com LES, ao contrário da população geral, é importante a dosagem conjunta dos anticorpos tireoidianos anti-TPO e anti-Tg. 
Resultados semelhantes foram encontrados por Vianna e cols., em 1991, (12) e Park e cols., em 1995, (15), os quais acrescentaram que os anticorpos tireoidianos podem ser bons preditores para alteração tireoidiana no LES (15). Vianna e cols. (12), em 1991, observaram prevalência de positividade de anti-Tg em $11 \%$ dos pacientes com LES e somente $2 \%$ nos controles. Resultado semelhante foi encontrado por Park e cols. (15), que observaram prevalência de $27 \%$ para anticorpos tireoidianos nos pacientes com LES, embora este estudo não tenha avaliado grupo-controle em paralelo.

Os resultados obtidos demonstram que alterações tireoidianas são mais prevalentes em pacientes com LES que na população geral e que, pelo fato de os sinais e sintomas das alterações tireoidianas serem inespecíficos, o diagnóstico clínico poderia ser feito tardiamente. A dosagem de TSH associada a dos anticorpos anti-TPO e antiTg são as mais indicadas no rastreamento das alterações tireoidianas em pacientes com LES, beneficiando pacientes já debilitados por uma doença crônica, com manifestações sistêmicas e seqüelas graves, por meio do diagnóstico precoce, possibilitando tratamento adequado.

Novos trabalhos são necessários para avaliar outros aspectos, como a evolução das alterações tireoidianas naqueles pacientes com anticorpos tireoidianos positivos, sem alteração da função tireoidiana.

\section{REFERÊNCIAS}

1. Lee SJ, Kavanaugh A. Autoimmunity, vasculitis and autoantibodies. J Allergy Clin Immunol. 2006;117 Suppl:445-50.

2. Scofield RH. Autoimmune thyroid disease in systemic lupus erythematosus and Sjögren's syndrome. Clin Exp Rheumatol. 1996;14:321-30.

3. Sato El, Bonfá ED, Costallat LTL, Silva NA, Brenol JCT, Santiago $\mathrm{MB}$, et al. Consenso brasileiro para o tratamento do lúpus eritematoso sistêmico (LES). Rev Bras Reumatol. 2002;42(6):362-70.

4. Montenegro RM, Montenegro Jr RM. Tiroidites - abordagem diagnóstico-terapêutica. In: Vilar L, editor. Endocrinologia clínica. $2^{\mathrm{a}}$ ed. Rio de Janeiro: MEDSI; 2001. p. 300-2.

5. Byron MA, Mowat AG. Thyroid disorders in systhemic lupus erythematosus. Ann Rheum Dis. 1987;46:579-83.

6. Weetman AP, Walport MJ. The association of autoimmune thyroiditis with systemic lupus erythematosus. Rheumatol Int. 1993;33:9-13.

7. Pyne $D$, Isenberg DA. Autoimmune thyroid disease in systemic lupus erythematosus. Ann Rheum Dis. 2002;61(1):70-2.

8. Tsai RT, Chang TC, Wang CR, Chuang CY, Chen CY. Thyroid disorders in chinese patients with systemic lupus erythematosus. Rheumatol Int. 1993;13:9-13.

9. Hijmans W, Doniach D, Roitt IM. Serological overlap between lupus erythematosus, rheumatoid arthritis and thyroid autoimmune disease. BMJ. 1961;508-12.
10. Goh KL, Wang F. Thyroid disorders in systemic lupus erythematosus. Ann Rheum Dis. 1986;45:579-83.

11. Miller FW, Moore GF, Weintraub BD, Steinberg AD. Prevalence of thyroid disease and abnormal thyroid function test results in patients with systemic lupus erythematosus. Artritis Rheum. 1987;30:1124-31.

12. Vianna JL, Haga HU, Asherson RA, Swana G, Hughes GRV. A prospective evaluation of antithyroid antibody prevalence in 100 patients with systemic lupus erythematosus. J Rheumatol. 1991;18:1193-5.

13. Magaro M, Zoli A, Altomonte L, Mirone L, La Sala L, Barini A, et al. The association of silent thyroiditis with active systemic lupus erythematosus. Clin Exp Rheumatol. 1992;10:67-70.

14. Arnaout MA, Nasrallah NS, EI-Khateeb MS. Prevalence of abnormal thyroid function tests in connective tissue disease. Scand J Rheumatol. 1994;23(3):128-32.

15. Park DJ, Cho CS, Lee SH, Park SH, Kim HY. Thyroid disorders in Korean patients with systemic lupus erythematosus. Scand J Rheumatol. 1995;24(1):13-7.

16. Kausman D, Isenberg DA. Thyroid autoimmunity in systemic lupus erythematosus: the clinical significance of a fluctuating course. Br J Rheumatol. 1995;34(4):361-4.

17. Boey ML, Fong PH, Lee JSC, Ng WY, Thai AC. Autoimmune thyroid disorders in SLE in Singapore. Lupus. 1993;2:51-4.

18. Kakehasi AM, Dias VN, Duarte JE, Lanna CCD, Moreira C, Carvalho MAP. Prevalência de disfunção tiroidiana no lúpus eritematoso sistêmico. Rev Bras Reumatol. 2000;40:269-274.

19. Biró et al. Association of systemic and thyroid autoimmune diseases. Clin Rheumatol. 2006;25:240-5.

20. Reuven M, Mishail S, Adawi IL, Luboshitzky R. Thyroid dysfunction in patient with systemic lupus erythematosus (SLE): relation to disease activity. Clin Rheumatol. 2007;26:1891-4.

21. Zuber M. Criteria for diagnosis and classification of connective tissue diseases. Rev Bras Rheumatol. 1997;37(1):47-52.

22. Baloch Z, Carayon P, Conte-Devolx B, Demers LM, Feldt-Rasmussen $U$, Henry JF, et al. Guidelines Committee, National Academy of Clinical Biochemistry (NACB). Laboratory medicine pactice guidelines (LMPG). Laboratory support for the diagnosis end monitoring of thyroid disease. Thyroid. 2003;13(1):3-126.

23. Larsen PR, Davies TF, Schlumberger MJ, Hay ID. Thyroid physiology and diagnostic evaluation of patients with thyroid disorders. In: Larsen PR, et al., editors. Williams textbook of endocrinology. $10^{\text {th }}$ ed. Philadelphia: Elsevier Science; 2003. p. 331-73.

24. Davies FT, Larsen PR. Thyrotoxicosis. In: Larsen PR, et al., editors. Williams textbook of endocrinology. $10^{\text {th }}$ ed. Philadelphia: Elsevier Science; 2003. p. 374-421.

25. Larsen PR, Davies TF. Hypothyroidism and Thyroiditis. In: Larsen PR, et al., editors. Williams textbook of endocrinology. $10^{\text {th }}$ ed. Philadelphia: Elsevier Science; 2003. p. 423-55.

26. Kakehasi AM, Dias VN, Duarte JE, Lanna CCD, Carvalho MAP. Alterações tiroidianas no lúpus eritematoso sistêmico: um estudo em 100 pacientes brasileiros. Rev Bras Reumatol. 2006;46(6):375-9.

Endereço para correspondência:

Daniela Pultrini Pereira de Oliveira Viggiano

Av. Quinta Radial, 79, ap. 202 - Setor Pedro Ludovico

74823-030, Goiânia GO

E-mail: danippo@ig.com.br 